\title{
ON THE EQUIVALENCE OF GAUSSIAN PROCESSES WITH FACTORABLE COVARIANCE FUNCTIONS
}

\author{
W. J. PARK
}

\begin{abstract}
Let $\left\{X_{t}\right\}_{t \in T}$ be a Gaussian process on a probability space $(\Omega, \mathscr{F}, P)$ with a factorable covariance function. We assume here that $T$ is a $p$-dimensional Euclidean space. The purpose of this paper is to give necessary and sufficient conditions that a probability measure $Q$ with respect to which $\left\{X_{t}\right\}_{t \in T}$ is a Gaussian process is equivalent to a probability measure $P$.
\end{abstract}

1. Introduction. Let $(\Omega, \mathscr{F}, P)$ be a probability space and let $T$ be the $p$-dimensional Euclidean space and $(T, \mathscr{B}, \mu)$ be the Lebesgue measure space. We denote by $Z(E, \omega)$ a Gaussian random measure on $(T, \mathscr{B}, \mu)$, i.e., it is a Gaussian set function satisfying

$$
\begin{gathered}
E[Z(E, \omega)]=\int_{\Omega} Z(E, \omega) d P(\omega)=0, \quad E \in \mathscr{B}, \\
E\left[Z(E, \omega) \cdot Z\left(E^{\prime}, \omega\right)\right]=\mu\left(E \cap E^{\prime}\right)
\end{gathered}
$$

for any $E$ and $E^{\prime}$ in $\mathscr{B} *=\{E \in \mathscr{B} ; \mu(E)<\infty\}$ (for its definition see Itô [4]).

Let $L^{2}(T)$ denote the Hilbert space of square integrable functions with respect to $\mu$. We shall consider a stochastic process $\left\{X_{t}\right\}_{t \in T}$ on $(\Omega, \mathscr{F}, P)$ which can be represented as

$$
X_{t}=\int_{T} \varphi(u, t) d Z(u, \omega)
$$

where $\varphi(\cdot, t) \in L^{2}(T)$ for each $t \in T$ and $\{\varphi(\cdot, t) ; t \in T\}$ spans $L^{2}(T)$. The process $\left\{X_{t}\right\}_{t \in T}$ so defined has the mean function identically equal to zero and its covariance function $R$ is clearly given by

$$
R(s, t)=E\left[X_{s} \cdot X_{t}\right]=\int_{T} \varphi(u, s) \varphi(u, t) d \mu(u) .
$$

Conversely a Gaussian process $\left\{X_{t}\right\}_{t \in \boldsymbol{T}}$ with zero mean function and the covariance function given by (1.4) can be represented in the form of (1.3) (see Cramer [3]).

Received by the editors May 3, 1971.

AMS 1970 subject classifications. Primary 60G15.

Key words and phrases. Gaussian random measure, equivalence of probability measures, covariance function, factorable.

(c) American Mathematical Society 1972 
The covariance function of the form (1.4) is called factorable (see Varberg [9]). The examples of Gaussian processes with factorable covariance functions are given in $\S 3$.

Let $\mathscr{F}_{X}$ be the $\sigma$-field generated by the process $\left\{X_{t}\right\}_{t \in T}$ and let $Q$ be a probability measure under which the process $\left\{X_{t}\right\}_{t \in T}$ is Gaussian with the mean function $m(t)$ and the covariance function $\Gamma_{Q}$. It is not necessarily required that $\Gamma_{Q}$ is factorable. We say that two probability measures $Q$ and $P$ are equivalent (designated as $Q \equiv P$ ) if they are mutually absolutely continuous. Our main result is the following theorem.

THEOREM. $Q \equiv P$ relative to $\mathscr{F}_{\mathrm{X}}$ if and only if there is a symmetric kernel $K \in L^{2}(T \times T)$ such that

$$
\begin{gathered}
\Gamma_{Q}(s, t)=R(s, t)-\int_{T \times T} \varphi(u, s) \varphi(v, t) K(u, v) d \mu(u) d \mu(v), \\
1 \notin \sigma(K),
\end{gathered}
$$

and there is a function $k \in L^{2}(T)$ such that

$$
m(t)=\int_{T} \varphi(u, t) k(u) d \mu(u),
$$

where $\sigma(K)$ denotes the spectrum of the operator given by

$$
(K f)(u)=\int_{T} K(s, u) f(s) d \mu(s), \quad f \in L^{2}(T) .
$$

2. Proof of Theorem. We will first develop necessary terminologies. Let $H(R)$ denote the reproducing kernel Hilbert space with the reproducing kernel $R$ and inner product $\langle,\rangle_{H(R)}$.

LEMMA 1. $H(R) \cong{ }^{J} L^{2}(T)$ with $J(R(\cdot, t))=\varphi(\cdot, t)$ and furthermore if $m \in H(R)$ and $J(m)=k$, then

$$
m(t)=\int_{T} \varphi(u, t) k(u) d \mu(u) .
$$

Here $H_{1} \cong{ }^{J} H_{2}$ for two Hilbert spaces $H_{1}$ and $H_{2}$ means that $J$ is a congruence between $H_{1}$ and $H_{2}$, i.e. inner product preserving isomorphism from $H_{1}$ to $H_{2}$.

The proof of the lemma is omitted since it can be easily shown by the similar method as the proof of Theorem 6 of Park [7] (only difference is to replace $1_{u}(\cdot)$ by $\left.\varphi(\cdot, u)\right)$.

Let $H(R) \otimes H(R)$ denote the tensor product of $H(R)$. Then it is again a reproducing kernel Hilbert space with the reproducing kernel $R\left(\cdot, t_{1}\right) R\left(*, t_{2}\right)$. Let us denote the element of $H(R) \otimes H(R)$ by $f \otimes g$ where $f$ and $g$ are in $H(R)$. (See Aronszajn [1].) 
If $h_{1} \otimes h_{2} \in H(R) \otimes H(R)$, then write

$$
\sigma\left(h_{1} \otimes h_{2}\right)=\frac{1}{2}\left[h_{1} \otimes h_{2}+h_{2} \otimes h_{1}\right] .
$$

The closed linear subspace of $H(R) \otimes H(R)$ spanned by elements of the type $\sum_{1}^{k} c_{n} \sigma\left(h_{1}^{n} \otimes h_{2}^{n}\right)$ is called the symmetric tensor product of $H(R)$, denoted by $\sigma(H(R) \otimes H(R))$, and it has as elements all symmetric functions in $H(R) \otimes H(R)$. The following lemma is immediate.

LEMMA 2. $\sigma(H(R) \otimes H(R)) \cong^{J_{1}} \sigma\left(L^{2}(T) \otimes L^{2}(T)\right)$ and if $F \in \sigma(H(R) \otimes H(R))$ and $J_{1}(F)=K$, then

$$
F(s, t)=\int_{T \times T} \varphi(u, s) \varphi(v, t) K(u, v) d \mu(u) d \mu(v) .
$$

It is noted that if $K \in \sigma\left(L^{2}(T) \otimes L^{2}(T)\right)$ then $K$ is symmetric and $K \in$ $L^{2}(T \times T)$. We will deduce our theorem using Theorem 1.3.1 of Oodaira [6], which states that $Q \equiv P$ relative to $\mathscr{F}_{X}$ if and only if

(2.1) $\Gamma_{Q}-R \in H(R) \otimes H(R)$,

(2.2) There are finite positive constants $c_{1}$ and $c_{2}$ such that $c_{1} R \ll$ $\Gamma_{Q} \ll c_{2} R$, and

(2.3) $m \in H(R)$.

It is clear that the conditions (1.7) and (2.3) are identical by Lemma 1 and so are the conditions (1.5) and $(2.1)$ by Lemma 2 (since $\Gamma_{Q}(\cdot, *)-$ $R(\cdot, *) \in \sigma(H(R) \otimes H(R)))$.

Let $\left\{\lambda_{j}\right\}$ and $\left\{g_{j}\right\}$ be eigenvalues and eigenfunctions of the operator $K$. Denote

$$
G_{j}(t)=\int_{T} \varphi(u, t) g_{j}(u) d \mu(u) .
$$

Then $G_{j} \in H(R)$ and it corresponds to $g_{j} \in L^{2}(T)$ under the congruence. Furthermore

$$
\begin{aligned}
\left\langle G_{j}, G_{j}\right\rangle_{H(R)} & =\left(g_{j}, g_{j}\right)=1 \\
\left\langle R(, t), G_{j}\right\rangle_{H(R)} & =G_{j}(t) \text { and } \\
\left\langle\Gamma_{Q}(\cdot, t), G_{j}\right\rangle_{H(R)} & =\left(\varphi(*, t)-(K \varphi(\cdot, t))(*), g_{j}\right)=\left(1-\lambda_{j}\right) G_{j}(t) .
\end{aligned}
$$

The last identity follows from the fact that, under the condition (1.5),

$$
\begin{aligned}
\Gamma_{Q}(\cdot, t) & =\int_{T} \varphi(u, \cdot) \varphi(u, t) d \mu(u)-\int_{T}\left[\int_{T} \varphi(u, \cdot) \varphi(v, t) K(u, v) d \mu(v)\right] d \mu(u) \\
& =\int_{T} \varphi(u, \cdot)\left[\varphi(u, t)-\int_{T} \varphi(v, t) K(u, v) d \mu(v)\right] d \mu(u) .
\end{aligned}
$$

Hence $\Gamma_{Q}(\cdot, t) \in H(R)$ for every $t \in T$ and it corresponds to $\varphi(\cdot, t)-$ $(K \varphi(*, t))(\cdot)$ in $L^{2}(T)$ under the congruence. 
Now $c_{1} R \ll \Gamma_{Q} \ll c_{2} R$ if and only if, according to (2.4), $c_{1} G_{j} \leqq\left(1-\lambda_{j}\right) G_{j} \leqq$ $c_{2} G_{j}$ for $j=1,2, \cdots$ if and only if $c_{1} \leqq 1-\lambda_{j} \leqq c_{2}$ for all $j$, i.e. $1-c_{2} \leqq \lambda_{j} \leqq$ $1-c_{1}<1$, i.e. $1 \notin \sigma(K)$. Therefore the conditions (2.2) and (1.6) are identical. This completes the proof.

3. Examples of Gaussian processes with factorable covariance functions. For the case that $T$ is the real line.

(A) Brownian motion:

$$
\begin{array}{rlrl}
R(s, t) & =\min (s, t), & \\
\varphi(s, t)=1_{t}(s) \text { where } 1_{t}(s)=1 & \text { if } s \leqq t \\
=0 & & \text { otherwise. }
\end{array}
$$

(B) Gaussian Markov process:

$$
\begin{array}{rlrl}
R(s, t) & =u(s) v(t) \quad \text { if } s \leqq t \\
& =u(t) v(s) \quad \text { if } t<s \\
\varphi(s, t) & =v(s)\left[\frac{d}{d t} \frac{u(t)}{v(t)}\right]^{1 / 2} \quad \text { if } s>t \text { or } s=t=0 \\
& =0 & & \text { otherwise. }
\end{array}
$$

(See Varberg [9] for the derivation of $\varphi$, and Beekman [2] for the examples of the process.)

For the case that $T$ is $p$-dimensional Euclidean space $E^{p}$.

(C) p-parameter Gaussian process:

$$
\begin{aligned}
& R(s, t)=\prod_{j=1}^{p} \min \left(s_{j}, t_{j}\right), \\
& \varphi(s, t)=\prod_{j=1}^{p} 1_{t_{j}}\left(s_{j}\right) \quad \text { for } s=\left(s_{1}, \cdots, s_{p}\right), t=\left(t_{1}, \cdots, t_{p}\right) .
\end{aligned}
$$

(See Park [7].)

(D) Brownian motion in Levy's sense: ( $p$ is odd)

$$
R(s, t)=\frac{1}{2}(|s|+|t|-|s-t|)
$$

where $|t|=\left(t_{1}^{2}+\cdots+t_{p}^{2}\right)^{1 / 2}$,

$$
\varphi(s, t)=\frac{1}{\sqrt{\Omega_{p+1}}}\left(e^{i(s, t)}-1\right)|s|^{-(p+1) / 2}
$$

where $\Omega_{p+1}$ is the area of the unit sphere in $E^{p+1}$. (See Molchan [5].) We remark that a proper modification of the theorem for the complex valued function $\varphi(s, t)$ can be easily seen.

For the case (A) and (C) the result is shown by Shepp [8] and Park [7] respectively. For the other case the theorem stated in this form is new as 
far as we know. It is also remarked that if $Q \equiv P$, then the Radon-Nikodym derivative $d Q / d P$ can be evaluated by a similar approach as Park [7], using the multiple Wiener integrals.

\section{REFERENCES}

1. N. Aronszajn, Theory of reproducing kernels, Trans. Amer. Math. Soc. 68 (1950), 337-404. MR 14, 479.

2. J. A. Beekman, Gaussian processes and generalized Schroedinger equations, J. Math. Mech. 14, (1965), 789-806. MR 31 \#4082.

3. H. Cramér, A contribution to the theory of stochastic processes, Proc. Second Berkeley Sympos. Math. Statist. and Probability (Berkeley, Calif., 1950), Univ. of California Press, Berkeley, Calif., 1951, pp. 329-339. MR 13, 475.

4. K. Itô, Multiple Wiener integral, J. Math. Soc. Japan 3 (1951), 157-169. MR 13, 364.

5. G. M. Molčan, Some problems connected with the Brownian motion of Lévy, Teor. Verojatnost. i Primenen. 12 (1967), 747-755=Theor. Probability Appl. 12 (1967), 682-690. MR 36 \#7206.

6. H. Oodaira, The equivalence of Gaussian stochastic process, Ph.D. Thesis, Michigan State University, East Lansing, Mich., 1963.

7. W. J. Park, A multi-parameter Gaussian process, Ann. Math. Statist. 41 (1970), 1582-1595.

8. L. A. Shepp, Radon-Nikodým derivatives of Gaussian measures, Ann. Math. Statist. 37 (1966), 321-354. MR 32 \#8408.

9. D. E. Varberg, On Gaussian measures equivalent to Wiener measure, Trans. Amer. Math. Soc. 113 (1964), 262-273. MR 29 \#2357.

Department of Mathematics, Wright State University, Dayton, Ohio 45431 